\title{
Sub-Gaussian short time asymptotics for measure metric Dirichlet spaces
}

\author{
András Telcs \\ Department of Computer Science and Information Theory, \\ University of Technology and Economics Budapest \\ Goldmann György tér 3, V2, 138 \\ Budapest, \\ H-1111, HUNGARY \\ telcs@szit.bme.hu
}

July 6, 2018

\begin{abstract}
This paper presents estimates for the distribution of the exit time from balls and short time asymptotics for measure metric Dirichlet spaces. The estimates cover the classical Gaussian case, the subdiffusive case which can be observed on particular fractals and further less regular cases as well. The proof is based on a new chaining argument and it is free of volume growth assumptions.
\end{abstract}

MSC2000 31C05, 60J45, 60J60

\section{Introduction}

The short-time asymptotics of the heat kernel for Riemannian manifolds has the classical form due to Varadhan [17]:

$$
\lim _{t \rightarrow 0} t \log p_{t}(x, y)=-\frac{1}{4} d^{2}(x, y) .
$$

It has got recently a lot of attention generalizing the short-time asymptotics to Dirichlet spaces. Such type of results obtained in Ramírez [12] and Hino, Ramírez [10]( see also Norris [11], Sturm [14]). The use of the intrinsic metric provides Gaussian estimates, $d^{2}(x, y)$ appear as in the case of $\mathbb{R}^{d}$ or Riemannian manifolds. A vast amount of papers was devoted to explore further 
properties of the heat kernel on Riemannian manifolds. Necessary and sufficient condition where provided for the local two-sided Gaussian estimate

$$
\frac{c}{V(x, \sqrt{t})} \exp \left(-\frac{d^{2}(x, y)}{c t}\right) \leq p_{t}(x, y) \leq \frac{1}{c V(x, \sqrt{t})} \exp \left(-\frac{c d^{2}(x, y)}{t}\right) \text {. }
$$

of the heat kernel $p_{t}(x, y)$ on Riemannian manifolds in Grigor'yan [6], SaloffCoste [13]. Here $V(x, r)$ stands for the volume (with respect. of the measure given on the space) of the ball $B(x, r)$ centered at $x$ with radius $r$. Similar but sub-Gaussian upper- and two-sided estimates have been obtained for particular fractals (see Barlow [3]). In [7] sufficient and necessary conditions were given for the sub-Gaussian estimates

$$
\begin{aligned}
& p_{t}(x, y) \geq \frac{1}{C V\left(x, t^{1 / \beta}\right)} \exp \left(-C\left(\frac{d^{\beta}(x, y)}{t}\right)^{\frac{1}{\beta-1}}\right) \\
& p_{t}(x, y) \leq \frac{1}{c V\left(x, t^{1 / \beta}\right)} \exp \left(-c\left(\frac{d^{\beta}(x, y)}{t}\right)^{\frac{1}{\beta-1}}\right)
\end{aligned}
$$

for weighted graphs and in [8] for measure metric spaces.

Consider $T_{x, R}$, the exit time from a ball $B(x, R)$. If the stating point $X_{0}=y$ let us denote the expected value of $T_{B(x, R)}$ by $\mathbb{E}_{y}(x, R)$ and by $E(x, R)$ if $y=x$. Let us denote by $a_{i} \simeq b_{i}$ the fact that there is a $c>0$ such that $\frac{1}{C}<\frac{a_{i}}{b_{i}}<C$ for all $i$. On many fractals (or fractal type graph) there is a space-time scaling function satisfying $\left(E_{F}\right)$ :

$$
E(x, R) \simeq F(R)
$$

in particular $\left(E_{\beta}\right)$ :

$$
E(x, R) \simeq R^{\beta}
$$

for a $\beta \geq 2$.

During the proof of the upper estimate an interesting side-result can be observed $\left(E_{\beta}\right)$ implies that

$$
\mathbb{P}\left(T_{x, R}<t \mid X_{0}=x\right) \leq C \exp \left(-\left(\frac{R^{\beta}}{C t}\right)^{\frac{1}{\beta-1}}\right) .
$$

One might wonder about the conditions which ensure similar lower bound or small time asymptotics for the heat kernel. The proof of the upper bound (1.6) uses some kind of chaining argument (cf. [3] and [7]). The off-diagonal 
lower estimates are typically shown using the chaining argument by Aronson [1] which uses a volume growth condition. In the present paper we provide lower counterpart of (1.6) and the short time asymptotics based on a new chaining argument ( Proposition 4.3 ). This chaining argument provides a lower estimate for the distribution of the hitting time of a ball, which we think might has some interest on its own. No volume growth or bounded covering conditions are needed.

In order to weaken the restriction on the mean exit time let us introduce some notions.

Definition 1.1 The sub-Gaussian kernels defined as follows. Let $A \subset M, t . R>$ 0 and $\kappa=\kappa_{A}(t, R)$ is the largest integer for which

$$
\frac{t}{\kappa} \leq q \inf _{y \in A} E\left(y, \frac{R}{\kappa}\right)
$$

in particular,

$$
\kappa(x, t, R)=\kappa_{B(x, R)}(t, R)
$$

and similarly $\nu=\nu_{A}(t, R)$ is the smallest for which

$$
\begin{gathered}
\frac{t}{\nu} \geq q \sup _{y \in A} E\left(y, \frac{R}{\nu}\right), \\
\nu(x, t, R)=\nu_{B(x, R)}(x, t, R) .
\end{gathered}
$$

We define $\kappa=0$ and $\nu=\infty$ if there is no such an integer. The constants $q>0$ will be specified later.

These integers will define the number of iterations we will use in chaining arguments which lead to the upper and lower estimates.

Our approach is different from those one [10]-[11] which use the intrinsic metric and recapture the usual Gaussian $R \rightarrow R^{2}$ scaling. We assume that the metric is given, predefined and we obtain a picture of the heat diffusion with respect to this metric. As Ramirez in [12] points out the two approaches are complement each other.

In the whole sequel we consider $(M, \mu, d)$ a locally compact separable measure metric space with Radon measure $\mu$, with full support. The metric is assumed to be a geodesic one. A strictly local regular Dirichlet form $(\mathcal{E}, \mathcal{F})$ in $L^{2}(M, \mu)$ is considered and let $\left(X_{t}\right)$ be the associated diffusion process on $M$ (cf. [5]). The corresponding Feller semigroup is $P_{t}$. Denote $\mathbb{P}_{x}, \mathbb{E}_{x}$ the probability measure and expectation given for $X_{0}=x \in M$. We assume that $\left(X_{t}\right)$ has a transition density $p_{t}(x, y)$ with respect to $\mu$, furthermore $p_{t}(x, y)$ satisfies the following property: 
(1.) $p_{t}(x, y) \geq 0$,

(2.) $\int_{M} p_{t}(x, y) d \mu(y)=1$,

(3.) $p_{t}(x, y)=p_{t}(y, x)$,

(4.) $p_{t}(x, y)=\int p_{s}(x, z) p_{t-s}(z, y) d \mu(z)$.

Now we give the definition of the elliptic Harnack inequality since it is a key condition in our main results.

Definition 1.2 A function $h: M \rightarrow \mathbb{R}$ said to be harmonic on an open set $A \subset M$ if it is defined on $\bar{A}$ and

$$
h(x)=E_{x}\left(h\left(X_{T_{A}}\right)\right) \quad \text { for all } x \in A .
$$

Definition 1.3 We will say that the elliptic Harnack inequality $(H)$ holds on $M$ if for all $x \in M, R>0$ and for any non-negative harmonic function $u$ which is harmonic in $B(x, 2 R)$, the following inequality holds

$$
\sup _{B(x, R)} u \leq H \inf _{B(x, R)} u
$$

with some constant $H \geq 1$ independent of $x$ and $R$.

The results of the present paper are the following.

Theorem 1.1 1. If there is a $C>0$ such that the condition $(\bar{E})$ holds, that is there is a $C>0$ such that

$$
\sup _{y \in B(x, R)} E_{y}(x, R) \leq C E(x, R)
$$

for all $x \in M, R>0$ then there is a $c>0$ such that for all $x \in M, t, R>0$

$$
\mathbb{P}_{x}\left(T_{x, R}<t\right) \leq \exp (-c \kappa(x, t, R))
$$

is true.

2. If $M$ satisfies the elliptic Harnack inequality, then there are $b, C>0$ such that for all $x \in M, t, R>0$

$$
\mathbb{P}_{x}\left(T_{x, R}<t\right) \geq \exp (-C \nu(x, t, b R)) .
$$

Theorem 1.2 Let us assume that there is an $R_{0}$ such that for all $r<R_{0}$

$$
E(x, r) \simeq r^{\beta}
$$


holds with a $\beta>1$. Let $A, B \subset M$ be measurable sets $0<\mu(A), \mu(B)<\infty$. Then we have the upper part of the short-time asymptotics:

$$
\lim _{t \rightarrow 0} t^{\frac{1}{\beta-1}} \log P_{t}(A, B) \leq-c[d(A, B)]^{\frac{\beta}{\beta-1}}
$$

and if in addition we assume that the $A, B$ sets are open and precompact furthermore the elliptic Harnack inequality holds then

$$
\lim _{t \rightarrow 0} t^{\frac{1}{\beta-1}} \log P_{t}(A, B) \geq-C[d(A, B)]^{\frac{\beta}{\beta-1}} .
$$

\section{Discussion}

The usual sub-diffusive picture can be recovered assuming that $E(x, R) \simeq$ $R^{\beta}$.

Corollary 2.1 Let us assume that $\left(E_{\beta}\right)$ holds on $M$ for a $\beta>1$

1. There are $c, C>0$ such that for all $x \in M, t, R>0, B=B(x, R)$

$$
\mathbb{P}_{x}\left(T_{x, R}<t\right) \leq C \exp \left(-c\left[\frac{R^{\beta}}{t}\right]^{\frac{1}{\beta-1}}\right)
$$

is true.

2. If $M$ satisfies the elliptic Harnack inequality, then there are $c, C>0$ such that

$$
P\left(T_{x, R}<t\right) \geq c \exp \left(-C\left[\frac{R^{\beta}}{t}\right]^{\frac{1}{\beta-1}}\right) .
$$

Problem 1 The classical bottle-neck construction shows that the condition $(\bar{E})$ does not imply the elliptic Harnack inequality. It would be interesting to find an example which satisfies the elliptic Harnack inequality but not $(\bar{E})$. At present we can not find such one.

There are nice examples where the diffusion speed is "direction" dependent (see [2] or [9]). We briefly recall one following [2]. Consider the direct product $M=\mathbb{R} \times S_{2}$, where $S_{2}$ stands for the Sierpinski gasket and let $Z_{t}=\left(X_{t}, Y_{t}\right)$ be the process on it, where $X_{t}$ is the standard Wiener process, and $Y_{t}$ is the anomalous diffusion process on $S_{2}$ (c.f. [3]) independent form $X_{t}$. It is clear that $X_{t}$ and $Y_{t}$ satisfy $(1.2),(1.3)$ with $\beta_{1}=2$ and $\beta_{2}>2$ respectively. Consequently the diagonal upper estimate holds for both and for $Z_{t}$ as well while the two-sided estimate is not true for any $\beta$. It is then clear that neither the elliptic Harnack inequality nor the short time asymtotics does hold. 


\section{Basic definitions}

The Dirichlet form can be restricted to a set $A$ acting only on functions with support in $A$. The corresponding process is simply killed on leaving $A$ (see $[5])$. Let us denote the associated heat kernel by $p_{t}^{A}(x, y)$ and the Green kernel by $g^{A}(x, y)$.

For sets we define

$$
d(A, B)=\inf _{x \in A, y \in B} d(x, y)
$$

To avoid technical difficulties we follow [12] and introduce

$$
P_{t}(A, B)=\int_{A} \int_{B} p_{t}(x, y) d \mu(y) d \mu(x) .
$$

Definition 3.1 We consider open metric balls defined by the metric d $(x, y)$ $x \in M, R>0$ as

$$
\begin{aligned}
B(x, R) & =\{y \in M: d(x, y)<R\} \\
S(x, R) & =\{y \in M: d(x, y)=R\} .
\end{aligned}
$$

Definition 3.2 The exit time from a set $A$ is defined as

$$
T_{A}=\inf \left\{t>0: X_{t} \in A^{c}\right\},
$$

its expected value is denoted by

$$
E_{x}(A)=\mathbb{E}\left(T_{A} \mid X_{0}=x\right),
$$

and we will use the $E=E(x, R)=E_{x}(x, R)=E_{x}(B(x, R))$ short notations.

Definition 3.3 The hitting time $\tau_{A}$ of a set $A$ is defined as the exit time of its complement:

$$
\tau_{A}=T_{A^{c}}
$$

and for $A=B(x, R)$ we use the shorter form $\tau_{x, r}$.

Definition 3.4 We introduce for a set $A \subset M$,

$$
\bar{E}(A)=\sup _{y \in A} E_{y}(A) .
$$

and for $x \in M, R>0$ we use the notation

$$
\bar{E}(x, R)=\bar{E}(B(x, R)) .
$$


Definition 3.5 For any sets $A, B$ the capacity is defined via the Dirichlet form $\mathcal{E}$ by

$$
\operatorname{cap}(A, B)=\inf \mathcal{E}(f, f),
$$

where the infimum runs for functions $f,\left.f\right|_{A}=1,\left.f\right|_{B}=0$. The resistance is defined as

$$
\rho(A, B)=\frac{1}{\operatorname{cap}(A, B)} .
$$

In particular we will use the following notations:

$$
\rho(x, r, R)=\rho\left(B(x, r), B^{c}(x, R)\right) .
$$

\section{Distribution of the exit time}

In this section we show Theorem 1.1. First we recall a result which was immediate from Lemma 5.3 of [15] for graphs and can be seen in the same way for the present setup (see also [3]).

Proposition 4.1 If we assume $(\bar{E})$ then there is a $c>0$ such that for all $x \in M, r>0, t \leq \frac{1}{2} E(x, r)$

$$
\mathbb{P}_{x}\left(T_{x, r}>t\right) \geq c .
$$

The proof of Theorem 1.1 based on the following observations. The probability of hitting a nearby ball in a "reasonable" time is bounded from below if the elliptic Harnack inequality holds.

Proposition 4.2 If the elliptic Harnack inequality $(H)$ holds then there are $c_{0}, c_{1}>0$ such that

$$
\mathbb{P}_{x}\left(\tau_{y, r}<s\right) \geq c_{0} .
$$

provided $\frac{1}{4} d(x, y) \leq r \leq d(x, y)$ and $s>\frac{2}{c_{1}} E(x, 9 r)$

At this point we specify the constant $q$ which appears in the definition of $\kappa, \nu$. Let $q=\frac{2}{c_{1}}$, which means, as we shall see in the Lemma 4.5, that it depends via $c_{1}$ on the constant of the Harnack inequality.

The key observation is the following proposition. It provides a lower bound for the probability hitting a ball in a given time. 
Proposition 4.3 If the elliptic Harnack inequality $(H)$ holds, then there are $b, C>0$ such that for all $x, y \in M, t>0, \frac{1}{4} d(x, y) \leq r<d=d(x, y)$,

$$
\mathbb{P}_{x}\left(\tau_{y, r}<t\right) \geq \exp -C \nu(x, t, b d) .
$$

First we need some lemmas.

Lemma 4.4 If the elliptic Harnack inequality $(H)$ holds then for $x \in M, R>$ $r>0, B=B(x, R), A=B(x, r)$

$$
\inf _{w \in \bar{A}} g^{B}(w, x) \simeq \rho(x, r, R) \simeq \sup _{w \in B \backslash A} g^{B}(w, x) .
$$

Proof. See Barlow's proof ([4], Proposition 2) which generalizes Propositions 4.1 and 4.3 of [7] where the additional hypothesis of bounded covering was used. Barlow's proof is given for weighted graphs, but word by word the same proof works in the continuous case.

Lemma 4.5 If $M$ satisfies the elliptic Harnack inequality $(H)$ then there is $a c_{1}>0$ such that for all $x \in M, r>0, w \in B=\bar{B}(x, 4 r), \frac{1}{4} d(w, x) \leq r$

$$
\mathbb{P}_{w}\left(\tau_{x, r}<T_{x, 5 r}\right)>c_{1} .
$$

Proof. The investigated probability

$$
u(w)=\mathbb{P}_{w}\left(\tau_{x, r}<T_{x, 5 r}\right)
$$

is the capacity potential between $B^{c}(x, 5 r)$ and $B(x, r)$ and clearly harmonic in $A=B(x, 5 r) \backslash B(x, r)$. So it can be decomposed in $A$

$$
u(w)=\int_{\bar{B}(x, r)} g^{B(x, 5 r)}(w, z) \pi(d z)
$$

where $\pi$ is the capacity measure with $\pi(\bar{B}(x, r))=1 / \rho(x, r, 5 r)$ with support in $\bar{B}(x, r)$. From the maximum (minimum) principle it follows that for any $z \in B(x, 2 r)$ the minimum of $g^{B(x, 5 r)}(w, z)$ in $w \in \bar{B}(x, 4 r)$ is attained at a $w \in S(x, 4 r)$, (and the same applies for $u(w)$ as well). It follows then from the Harnack inequality for $g^{B(x, 5 r)}(w,$.$) in B(x, 2 r)$ that for any $w \in S(x, 4 r)$

$$
\begin{gathered}
\inf _{z \in \bar{B}(x, 2 r)} g^{B(x, 5 r)}(w, z) \geq c g^{B(x, 5 r)}(w, x) \\
u(w)=\int_{\bar{B}(x, r)} g^{B(x, 5 r)}(w, z) \pi(d z) \geq \frac{c g^{B(x, 5 r)}(w, x)}{\rho(x, r, 5 r)}
\end{gathered}
$$


From Lemma 4.4 we know that

$$
\sup _{y \in B(x, 5 r) \backslash B(x, 4 r)} g^{B(x, 5 r)}(y, x) \simeq \rho(x, 4 r, 5 r) \simeq \inf _{w \in \bar{B}(x, 4 r)} g^{B(x, 5 r)}(w, x) .
$$

which means that

$$
u(w) \geq c \frac{\rho(x, 4 r, 5 r)}{\rho(x, r, 5 r)}
$$

Similarly from Lemma 4.4 it follows that

$$
\sup _{y \in B(x, 5 r) \backslash B(x, r)} g^{B(x, 5 r)}(v, x) \simeq \rho(x, r, 5 r) \simeq \inf _{w \in \bar{B}(x, r)} g^{B(x, 5 r)}(w, x) .
$$

Finally if $y_{0} \in S(x, r)$ is on the ray from $x$ to $y \in S(x, 4 r)$ then iterating the Harnack inequality along a finite chain of balls of radius $r / 4$ along this ray from $y_{0}$ to $y$ one obtains

$$
g^{B(x, 5 r)}(y, x) \simeq g^{B(x, 5 r)}\left(y_{0}, x\right)
$$

which results that

$$
\rho(x, r, 5 r) \leq c \rho(x, 4 r, 5 r),
$$

and the statement follows from (4.18).

Proof of Proposition 4.2. We insert the exit time $T_{x, 9 r}$ into the inequality $\tau_{y, r}<t$

$$
\begin{aligned}
\mathbb{P}_{x}\left(\tau_{y, r}<t\right) & \geq \mathbb{P}_{x}\left(\tau_{y, r}<T_{x, 9 r}<t\right) \\
& =\mathbb{P}_{x}\left(\tau_{y, r}<T_{x, 9 r}\right)-\mathbb{P}_{x}\left(\tau_{y, r}<T_{x, 9 r}, T_{x, 9 r} \geq t\right) \\
& \geq \mathbb{P}_{x}\left(\tau_{y, r}<T_{x, 9 r}\right)-\mathbb{P}_{x}\left(T_{x, 9 r} \geq t\right)
\end{aligned}
$$

On one hand the Markov inequality results that

$$
\mathbb{P}_{x}\left(T_{x, 9 r} \geq t\right) \leq \frac{E(x, 9 r)}{t} \leq \frac{E(x, 9 r)}{\frac{2}{c_{1}} E(x, 9 r)}<c_{1} / 2
$$

and on the other hand $B(y, 5 r) \subset B(x, 9 r)$, hence

$$
\mathbb{P}_{x}\left(\tau_{y, r}<T_{x, 9 r}\right) \geq \mathbb{P}_{x}\left(\tau_{y, r}<T_{y, 5 r}\right)
$$

and Lemma 4.5 can be applied to get

$$
\mathbb{P}_{x}\left(\tau_{y, r}<T_{y, 5 r}\right) \geq c_{1}
$$

The result follows with $c_{0}=c_{1} / 2$. 
Lemma 4.6 For all $x \neq y \in M, t>0, l>1$

$$
\mathbb{P}_{x}\left(\tau_{y, r}<t\right) \geq \inf _{z, w \in \bar{B}(x, d), d(z, w) \leq 4 r}\left[\mathbb{P}_{z}\left(\tau_{w, r}<s\right)\right]^{l},
$$

where $d=d(x, y), s=\frac{t}{l}, r=\frac{d}{3 l}$.

Proof. Let us consider a geodesic path $\pi$ from $x$ to $y$ Let $x_{1} \in \pi$ such that $d\left(x, x_{1}\right)=3 r, x_{2} \in \pi$ with $d\left(x_{1}, x_{2}\right)=3 r$ etc. and finally $x_{l}=y$. Let $\tau_{i}=\tau_{x_{i}, r}, \tau_{0}=0$ and $A_{i}=\left\{\tau_{i}-\tau_{i-1}<s\right\}$ for $i=1 \ldots l, \tau_{0}=0$. Denote $A_{i}(z)=\left\{\tau_{i}-\tau_{i-1}<s, X_{\tau_{i}}=z\right\}$ and $\zeta_{i}=X_{\tau_{i}}$. One can observe that $\prod_{i=0}^{l} A_{i}$ means that the process spends less than time $s$ between the first hit of the consecutive $B_{i}=B\left(x_{i}, r\right)$ balls, consequently

$$
\begin{gathered}
\tau_{y, r}=\tau_{l}=\sum_{i=1}^{l} \tau_{i}-\tau_{i-1}, \\
\left\{\tau_{y, r}<t\right\}=\left\{\sum_{i=1}^{l} \tau_{i}-\tau_{i-1}<t\right\} \supset \prod_{i=0}^{l-1} A_{i} .
\end{gathered}
$$

Let us continue with the following estimates.

$$
\begin{aligned}
\mathbb{P}_{x}\left(\tau_{y, r}<t\right) & \geq \mathbb{P}_{x}\left(\prod_{i=0}^{l} A_{i}\right)=\mathbb{E}_{x}\left(A_{l}\left(\zeta_{l}\right) \prod_{i=0}^{l-1} A_{i}\right) \\
& =\mathbb{E}_{x}\left(I\left(A_{l}\right) \mid \prod_{i=0}^{l-1} I\left(A_{i}\right)\right) \mathbb{P}_{x}\left(\prod_{i=0}^{l-1} I\left(A_{i}\right)\right)
\end{aligned}
$$

Now we use the strong Markov property (c.f [5] (A.2.3. $\left.)^{\prime}\right)$ to obtain the following.

$$
\begin{aligned}
& \mathbb{E}_{x}\left(\mathbb{E}_{\zeta_{l-1}}\left[I\left(A_{l}\right)\right] \mid \prod_{i=0}^{l-1} I\left(A_{i}\right)\right) \mathbb{P}_{x}\left(\prod_{i=0}^{l-1} I\left(A_{i}\right)\right) \\
\geq & \inf _{z \in S\left(x_{l-1}, r\right)} \mathbb{P}_{z}\left(A_{l}\right) \mathbb{P}_{x}\left(\prod_{i=0}^{l-1} I\left(A_{i}\right)\right)
\end{aligned}
$$

Continuing the iteration one obtains

$$
\begin{aligned}
& \mathbb{P}_{x}\left(\tau_{y, r}<t\right) \\
\geq & \inf _{z \in S\left(x_{l-1}, r\right)} \mathbb{P}_{z}\left(A_{l}\right) \mathbb{P}_{x}\left(\prod_{i=1}^{l-1} I\left(A_{i}\right)\right) \\
\geq & \inf _{z, w \in B(x, d), d(z, w) \leq 4 r}\left[\mathbb{P}_{z}\left(\tau_{w, r}<s\right)\right]^{l} .
\end{aligned}
$$


Proof of Proposition 4.3. Let us apply Lemma 4.6 with $l=\nu_{B}(t, 3 d)$. One should observe that we have a uniform constant lower bound for $\mathbb{P}_{z}\left(\tau_{w, r}<s\right)$ by Proposition 4.2 provided

$$
s>\frac{2}{c_{1}} E(z, 9 r) .
$$

This condition is ensured by the definition of $\nu_{B}(t, 3 d)$ with $s=\frac{t}{\nu}, r=\frac{d}{3 l}$ since $9 r=\frac{3 d}{l}$. Finally from $l=\nu_{B(x, d)}(t, 3 d) \leq \nu_{B(x, 3 d)}(t, 3 d)=\nu(x, t, 3 d)$ follows the statement.

Proof of Theorem 1.1. The upper estimate of Theorem 1.1 can be seen along the lines of the proof of Theorem 5.1 in [15]. The lower bound is immediate from (4.14). Let $b=6$ then for any $y \in S(x, 2 R), r=\frac{6 R}{l}, l=$ $\nu(x, t, 6 R)$

$$
\mathbb{P}_{x}\left(T_{x, R}<t\right) \geq \mathbb{P}_{x}\left(\tau_{y, r}<t\right)
$$

and the result follows from Proposition 4.3.

\section{Short time asymptotics}

Before we start the proof let us make it clear why we assume that $E(x, r) \simeq$ $r^{\beta}$ for $r<R_{0}$. Without this assumption the upper and lower estimates will not meet. Using the proof which will follow, one can obtain separate upper and lower bound for the short time asymptotics. Let us mention that as $t$ goes to zero the functions $\kappa$ and $\nu$ go to infinity (c.f. Definition 1.1) consequently for enough small $t \frac{r}{\kappa}$ and $\frac{r}{\nu}$ fall below $R_{0}$ and the polynomial approximation can be used and provides us the more readable and coinciding upper and lower bound.

Proof of Theorem 1.2. Consider $A, B \subset M$, denote $d=d(A, B)$ and let us use (2.10) to get

$$
\begin{aligned}
P_{t}(A, B) & =\int_{A}\left(P_{t} 1_{B}\right)(x) d \mu(x) \\
& \leq C \mu(A) \mu(B) \exp \left[-c\left(\frac{d^{\beta}}{t}\right)^{\frac{1}{\beta-1}}\right],
\end{aligned}
$$

which results that

$$
t^{\frac{1}{\beta-1}} \log P_{t}(A, B) \leq t^{\frac{1}{\beta-1}}[C+\log \mu(A)+\log \mu(B)]-c d(A, B)^{\frac{\beta}{\beta-1}},
$$

and

$$
\lim _{t \rightarrow 0} t^{\frac{1}{\beta-1}} \log \left(P_{t}(A, B)\right) \leq-c d(A, B)^{\frac{\beta}{\beta-1}}
$$


If $A, B$ are open we can find for any $x \in A, y \in B$ a very small $r$ such that the balls $A_{r}=B(x, r) \subset A, B_{r}=B(y, r) \subset B$. For the lower estimate we decompose the path with the first hit of $B(y, r / 2) \subset B(y, r) \subset B$. The only task is to show that the probability that the process stays in $B(y, r)$ until $t$ is bounded from below by a constant. Let us observe that

$$
R=d(x, y) \geq d(A, B)+2 r .
$$

Denote $\xi=X_{\tau}$, where $\tau=\tau_{y, r / 2}$,

$$
P_{t}(A, B)=\int_{A} P_{t}(x, B) d \mu(x) \geq \int_{A} P_{t}\left(x, B_{r}\right) d \mu(x) .
$$

For any fixed $x \in A$

$$
P_{t}\left(x, B_{r}\right) \geq \mathbb{E}_{x}\left(I(\tau<t) I\left(X_{t} \in B_{r}\right)\right)
$$

Now we use the strong Markov property(c.f [5] (A.2.3. $\left.)^{\prime}\right)$ to decompose the path according to $\xi=X_{\tau}$ and obtain

$$
\begin{aligned}
& \mathbb{E}_{x}\left(I(\tau<t) I\left(X_{t} \in B_{r}\right)\right) \\
= & \mathbb{E}_{x}\left(I(\tau<t) \mathbb{E}_{\xi}\left[I\left(X_{t-\tau} \in B_{r}\right)\right]\right) \\
\geq & \mathbb{E}_{x}\left(I(\tau<t) \mathbb{E}_{\xi}\left[I\left(T_{y, r}>t-\tau\right)\right]\right) \\
\geq & \mathbb{E}_{x}\left(I(\tau<t) \mathbb{E}_{\xi}\left[I\left(T_{\xi, r / 2}>t\right)\right]\right) \\
\geq & \mathbb{E}_{x}\left(I(\tau<t) \inf _{w \in S(y, r / 2)} \mathbb{E}_{w}\left[I\left(T_{w, r / 2}>t\right)\right]\right),
\end{aligned}
$$

It is clear from (4.12) that for all $w$

$$
\mathbb{P}_{w}\left(T_{w, r / 2}>t\right) \geq c^{\prime}
$$

if

$$
t<\frac{1}{2} E(w, r / 2) .
$$

This can be ensured using the lower bound of $\left(E_{\beta}\right)$ for small $t$ :

$$
t<\frac{c}{2}\left(\frac{r}{2}\right)^{\beta}<\frac{1}{2} E(w, r / 2) .
$$

So if (5.19) holds we have

$$
\begin{aligned}
P_{t}(x, B(y, r)) & \geq c^{\prime} \mathbb{E}_{x}(I(\tau<t)) \\
& =c^{\prime} \mathbb{P}_{x}(\tau<t) .
\end{aligned}
$$


Set $\rho=\varepsilon r, B^{\prime}=B(y, \rho)$. The next step is to use (4.14) to get

$$
\mathbb{P}_{x}(\tau<t) \geq \exp \left(-C\left(\frac{R^{\beta}}{t}\right)^{\frac{1}{\beta-1}}\right) .
$$

The proper choice of the constants follows from the restrictions:

$$
\begin{gathered}
\varepsilon r=\rho, \varepsilon<1 / 2, \\
\frac{t}{k} \simeq \rho^{\beta}=\left(\frac{R}{3 k}\right)^{\beta},
\end{gathered}
$$

and

$$
t^{\frac{1}{\beta}}<c r
$$

If we consider

$$
\rho=\frac{R}{3 k}=\varepsilon r
$$

it follows that the proper choice for $\varepsilon$ is

$$
\varepsilon=c\left(\frac{r}{R}\right)^{\frac{1}{\beta-1}}
$$

The short time asymptotics now is immediate.

$$
\begin{gathered}
P_{t}(A, B) \geq \mu(A) c \exp \left(-C\left(\frac{R^{\beta}}{t}\right)^{\frac{1}{\beta-1}}\right), \\
\lim _{t \rightarrow 0} t^{\frac{1}{\beta-1}} \log P_{t}(A, B) \geq-C d^{\frac{\beta}{\beta-1}}(x, y) .
\end{gathered}
$$

Finally we let $d(x, y) \rightarrow d(A, B)$ and we receive the lower bound.

\section{References}

[1] Aronson D.G. Non-negative solutions of linear parabolic equations. Ann. Scuola Norm. Sup. Pisa cl. Sci (3) 22 (1968) 607-694; Addendum 25 (1971), 221-228.

[2] Barlow M.T., Which values of the volume growth and escape time exponent are possible for a graph?, Revista Mat. Iberoamericana 20 (2000) $1-31$ 
[3] Barlow, M.T., St Flour Lecture Notes: Diffusions on Fractals. In: Lect. Notes Math. 1690 .

[4] Barlow M.T., Some remarks on the elliptic Harnack inequality, preprint

[5] Fukushima, M., Oshima, Y., Takeda M. Dirichlet forms and symmetric Markov Processes de Gruyter Studies in Mathematics, 19. Walter de Gruyter \& Co., Berlin, 1994.

[6] Grigor'yan A., The heat equation on non-compact Riemannian manifolds, Mat. Sbornik, 182, 1991,55-87, Engl. Transl. Math. USSR Sb, 72, (1992) 47-77

[7] Grigor'yan A., Telcs A., Harnack inequalities and sub-Gaussian estimates for random walks, Math. Annalen 324 (2002) 521-55

[8] Grigor'yan A., Telcs A., Heat kernel estimates on measure metric spaces, in preparation

[9] Hambly, B.M., Kumugai, T., Heat kernel estimates for symmetric random walks on a class of fractal graphs and stability under rough isometry, Proc. of Symposia in Pure Math. 72, Part 2, pp. 233-260, Amer. Math. Soc. 2004.

[10] Hino M., Ramírez J.A., Small-time Gaussian Behavior of Symmetric Diffusion Semigroups, Annals of probability (2003) 15:23

[11] Norris J.R., Heat kernel asymptotics and the distance function in Lipschitz Riemannian manifolds, Acta Math. 179 (1997) 79-103

[12] Ramírez J.A., Short-time Asymptotics in Dirichlet spaces, Comm. Pure Appl. Math. 54 (2001) 259-293

[13] Saloff-Coste L., A note on Poincaré, Sobolev and Harnack inequalities, Duke Math. J., IMRN, 2, (1992) 27-38

[14] Sturm K-T., Analysis on local Dirichlet spaces III. The parabolic Harnack inequality, J. Math. Pures. Appl. (9) 75 (1996) no.3. 273-297

[15] Telcs A., Volume and time doubling of graphs and random walks, the strongly recurrent case, Comm. Pure and Appl. Math., Volume 54, Issue 8 (2001) 975-1018

[16] Telcs A., Random walks on graphs with volume and time doubling, submitted 
[17] Varadhan, S.R.S., On the behavior of the fundamental solution of the heat equation with variable coefficients. Comm. Pure Appl. Math. 20 (1967) 431-455 